\title{
Learning to Customize Model Structures for Few-shot Dialogue Generation Tasks
}

\author{
Yiping Song ${ }^{1}$, Zequn Liu ${ }^{1}$, Wei Bi ${ }^{2}$, Rui Yan ${ }^{3}$, Ming Zhang ${ }^{1 *}$ \\ ${ }^{1}$ Department of Computer Science, School of EECS, Peking University, Beijing, China \\ ${ }^{2}$ Tencent AI Lab, Shenzhen, China \\ ${ }^{3}$ Wangxuan Institute of Computer Technology, Peking University, Beijing, China \\ songyiping, zequnliu, mzhang_cs, ruiyanepku.edu.cn \\ victoriabiatencent.com
}

\begin{abstract}
Training the generative models with minimal corpus is one of the critical challenges for building open-domain dialogue systems. Existing methods tend to use the meta-learning framework which pre-trains the parameters on all non-target tasks then fine-tunes on the target task. However, fine-tuning distinguishes tasks from the parameter perspective but ignores the model-structure perspective, resulting in similar dialogue models for different tasks. In this paper, we propose an algorithm that can customize a unique dialogue model for each task in the few-shot setting. In our approach, each dialogue model consists of a shared module, a gating module, and a private module. The first two modules are shared among all the tasks, while the third one will differentiate into different network structures to better capture the characteristics of the corresponding task. The extensive experiments on two datasets show that our method outperforms all the baselines in terms of task consistency, response quality, and diversity.
\end{abstract}

\section{Introduction}

Generative dialogue models often require a large amount of dialogues for training, and it is challenging to build models that can adapt to new domains or tasks with limited data. With recent advances in large-scale pre-training [Peters et al., 2018; Howard and Ruder, 2018; Radford et al., 2018; Devlin et al., 2018], we can first pre-train a generative model on large-scale dialogues from the non-target domains and then fine-tune on the task-specific data corpus [Wang et al., 2019a; Alt et al., 2019a; Klein, 2019]. While pre-training is beneficial, such models still require sufficient taskspecific data for fine-tuning. They cannot achieve satisfying performance when very few examples

\footnotetext{
${ }^{*}$ Corresponding author
}

are given [Bansal et al., 2019]. Unfortunately, this is often the case in many dialogue generation scenarios. For example, in personalized dialogue generation, we need to quickly adapt to the response style of a user's persona by just a few his or her dialogues [Madotto et al., 2019; Zhang et al., 2018]; in emotional dialogue generation, we need to generate a response catering to a new emoji using very few utterances containing this emoji [Zhou et al., 18; Zhou and Wang, 2018]. Hence, this is the focus of our paper - few-shot dialogue generation, i.e. training a generative model that can be generalized to a new task (domain) within $k$-shots of its dialogues.

A few works have been proposed to consider few-shot dialogue generation as a meta-learning problem [Madotto et al., 2019; Qian and Yu, 2019; Mi et al., 2019]. They all rely on the popular modelagnostic meta-learning (MAML) method [Finn et al., 2017]. Take building personalized dialogue models as an example, previous work treats learning dialogues with different personas as different tasks [Madotto et al., 2019; Qian and Yu, 2019]. They employ MAML to find an initialization of model parameters by maximizing the sensitivity of the loss function when applied to new tasks. For a target task, its dialogue model is obtained by finetuning the initial parameters from MAML with its task-specific training samples.

Despite the apparent success in few-shot dialogue generation, MAML still has limitations [Zintgraf et al., 2019]. The goal of generative dialogue models is to build a function mapping a user query to its response, where the function is determined by both the model structure and parameters [Brock et $a l ., 2018]$. By fine-tuning with a fixed model structure, MAML only searches the optimal parameter settings in the parameter optimization perspective but ignores the search of optimal network structures in the structure optimization perspective. More- 
over, language data are inherently discrete and dialogue models are less vulnerable to input changes than image-related models [Niu and Bansal, 2018], which means gradients calculated from a few sentences may not be enough to change the output word from one to another. Thus there is a need to develop an effective way to adjust MAML for large model diversity in dialogue generation tasks.

In this paper, we propose the Customized Model Agnostic Meta-Learning algorithm (CMAML) that is able to customize dialogue models in both parameter and model structure perspective under the MAML framework. The dialogue model of each task consists of three parts: a shared module to learn the general language generation ability and common characteristics among tasks, a private module to model the unique characteristic of this task, and a gate to absorb information from both shared and private modules then generate the final outputs. The network structure and parameters of the shared and gating modules are shared among all tasks, while the private module starts from the same network but differentiates into different structures to capture the task-specific characteristics.

In summary, our contributions are as follows:

- We propose the CMAML algorithm that can customize dialogue models with different network structures for different tasks in the few-shot setting. The algorithm is general and well unified to adapt to various few-shot generation scenarios.

- We propose a pruning algorithm that can adjust the network structure for better fitting the training data. We use this strategy to customize unique dialogue models for different tasks.

- We investigate two crucial impact factors for meta-learning based methods, i.e., the quantity of training data and task similarity. We then describe the situations where the meta-learning can outperform other fine-tuning methods.

\section{Related Work}

Few-shot Dialogue Generation. The past few years have seen increasing attention on building dialogue models in few-shot settings, such as personalized chatbots that can quickly adapt to each user's profile or knowledge background [Zhang et al., 2018; Madotto et al., 2019], or that respond with a specified emotion [Zhou et al., 18; Zhou and Wang, 2018]. Early solutions are to use explicit [Tian et al., 2017; Zhang et al., 2018; Zhou et al., 18] or implicit [Li et al., 2016b; Zhou and
Wang, 2018; Zhou et al., 18] task descriptions, then introduce this information into the generative models. However, these methods require manually created task descriptions, which are not available in many practical cases.

An alternative promising solution to building few-shot dialogue models is the meta-learning methods, especially MAML [Finn et al., 2017]. Madotto et al. (2019) propose to regard learning with the dialogue corpus of each user as a task and endow the personalized dialogue models by fine-tuning the initialized parameters on the taskspecific data. Qian and Yu (2019) and Mi et al. (2019) treat the learning from each domain in multidomain task-oriented dialogue generation as a task, and apply MAML in a similar way. All these methods do not change the original MAML but directly apply it to their scenarios due to the model-agnostic property of MAML. Thus, task differentiation always counts on fine-tuning, which only searches the best model for each task at the parameter level but not the model structure level.

Meta-learning. Meta-learning has achieved promising results in many NLP problems recently due to its fast adaptation ability on a new task using very few training data [Yu et al., 2019; Wang et al., 2019b; Obamuyide and Vlachos, 2019b; Alt et al., 2019b]. In general, there are three categories of meta-learning methods: metric-based methods [Vinyals et al., 2016; Snell et al., 2017; Sung et al., 2018; Ye and Ling, 2019] which encode the samples into an embedding space along with a learned distance metric and then apply a matching algorithm, model-based methods [Santoro et al., 2016; Obamuyide and Vlachos, 2019a] which depend on the model structure design such as an external memory storage to facilitate the learning process, and optimization-based methods [Finn et al., 2017; Andrychowicz et al., 2016; Huang et al., 2018] which learn a good network initialization from which fine-tuning can converge to the optimal point for a new task with only a few examples. Methods belonging to the first two are proposed for classification, and those in the third category are model-agnostic. Therefore, it is intuitive to apply the optimization-based methods, in which MAML is most popular, for dialogue generation tasks.

However, some researchers found that the original MAML has limited ability to model taskspecific characteristics in the image or text classification scenarios [Jiang et al., 2018; Sun et al., 


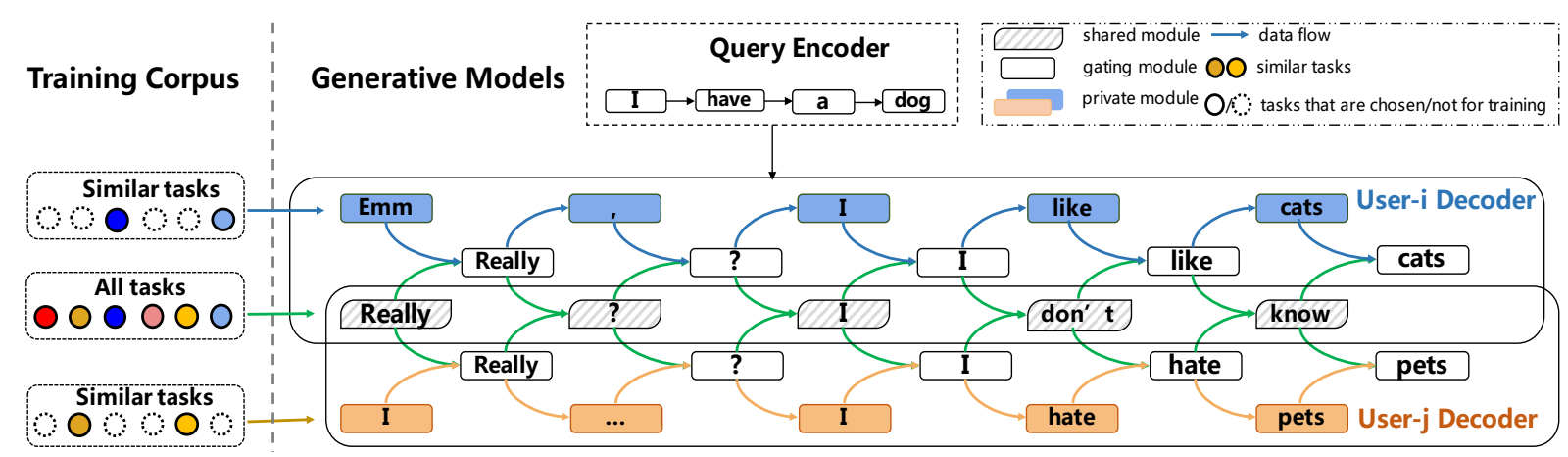

Figure 1: The proposed CMAML algorithm applying on the personalized dialogue systems. Each customized dialogue model Seq2SPG consists of a shared, a private, and a gating module. The shared and gating module are the same among users and are trained on all tasks. The private module is unique for each user to describe this user's persona, and is trained on the corresponding and similar tasks. The lines in color indicate the data flow directions.

2019; Liu et al., 2020]. Jiang et al. (2018) build an attention layer over the convolutional layers, where the convolutional layer is for general features and the attention layer is for task-specific features. Sun et al. (2019) propose to learn a task-specific shifting and scaling operation on the general shared feed-forward layers. However, the involved operations in these two methods such as shifting and scaling are designed for feed-forward networks, and can not be applied to the generative models which generally rely on Seq2seq [Sutskever et al., 2014] models with recurrent GRU [Cho et al., 2014] or LSTM [Hochreiter and Schmidhuber, 1997] cells. In this paper, we propose a new meta-learning algorithm based on MAML that can enhance task-specific characteristics for generation models.

\section{Dialogue Model}

In this section, we firstly describe the network structure of the proposed dialogue model, and then briefly introduce its pre-training.

\subsection{Model Architecture}

We aim to build dialogue models for different generation tasks in the few-shot setting. Now, we first describe the dialogue model of each task to be used in our training algorithm. It involves three network modules and noted as Seq2SPG (in Figure 1):

Shared Module. It gains the basic ability to generate a sentence and thus its parameters are shared among all tasks. We employ a prevailing Seq2seq dialogue model [Bahdanau et al., 2014]. At each decoding step $t$, we feed the word $x_{t}$ and last hidden state $h_{t-1}$ to the decoding cell, and obtain an output distribution $o_{s}$ over the vocabulary.
Private Module. It aims at modeling the unique characteristics of each task. We design a multilayer perception (MLP) in the decoder to fulfill this goal. Each task has its unique MLP network, which starts from the same initialization and then evolves into different structures during training. At each decoding step $t$, the MLP takes the word $x_{t}$ and the output $h_{t-1}$ of the shared module at step $t-1$ as input, then outputs a distribution $o_{p}$ over the vocabulary. In our experiments, we also explore different inputs for the private module.

Gating Module. We use a gate to fuse information from the shared and private modules:

$$
\begin{aligned}
g_{s} & =\tanh \left(W_{s}\left[o_{s}, o_{p}\right]+b_{s}\right) \\
g_{p} & =\tanh \left(W_{p}\left[o_{s}, o_{p}\right]+b_{p}\right) \\
o & =g_{s} \circ o_{s}+g_{p} \circ o_{p}
\end{aligned}
$$

where $W_{s}, W_{p}, b_{s}, b_{p}$ are parameters, $\circ$ is elementwise product, and $o$ is the word distribution.

\subsection{Training Overview}

For the rest of the paper, $p(\mathcal{T})$ denotes the task distribution, $T_{i}$ denotes the $i$-th task to be trained, $D_{i}^{\text {train }}$ and $D_{i}^{\text {valid }}$ denotes the training and validation corpus of task $T_{i}$, and $\theta_{i}$ denotes all training parameters of the dialogue model for $T_{i}$, which include parameters $\theta^{s} / \theta_{i}^{p} / \theta^{g}$ in the shared/private/gating module respectively. we consider a model represented by a parameterized function $f$ with parameters $\theta$. The model training for all tasks consists of two steps: pre-training and customized model training.

In pre-training, CMAML employs the vanilla MAML to obtain a pre-trained dialogue model as the initial model $\theta$ for all tasks. At the beginning of the MAML, $\theta$ are randomly initialized. Then, two 
main procedures perform iteratively: meta-training and meta-testing. In meta-training, MAML first samples a set of tasks $T_{i} \sim p(\mathcal{T})$. Then, for each task $i$, MAML adapts $\theta$ to get $\theta_{i}^{\prime}$ with the taskspecific data, which is,

$$
\theta_{i}^{\prime}=\theta-\alpha \nabla_{\theta} \mathcal{L}_{D_{i}^{\text {train }}}(f(\theta))
$$

In the meta-testing, MAML tests tasks $T_{i} \sim p(\mathcal{T})$ with $\theta_{i}^{\prime}$ to obtain the losses and then updates $\theta$ by

$$
\theta=\theta-\beta \nabla_{\theta} \sum_{T_{i} \sim p(\mathcal{T})} \mathcal{L}_{D_{i}^{\text {valid }}}\left(f\left(\theta_{i}^{\prime}\right)\right)
$$

Here, $\alpha$ and $\beta$ are hyper-parameters.

In standard MAML, each task obtains its parameters $\theta_{i}$ by fine-tuning the pre-trained $\theta$. However, recall that fine-tuning fails to search the best model in the network structure perspective. Also, the generative models are less vulnerable to input changes, thus a few utterances may not be enough to adapt $\theta$ into diverse $\theta_{i}$ for different tasks. To address these issues, we do not perform direct fine-tuning on each task, but design our second training step - Customized Model Training, in which the pretrained private module can evolve into different structures to capture the characteristics of each task and encourage model diversity.

\section{Customized Model Training}

After obtaining the pre-trained model $\theta$ from MAML, we employ Customized Mode Training with the following two updating steps:

- Private Network Pruning. This step is applied for the private module only, which is to differentiate the MLP structure of each task. Each task has a different MLP structure by retaining its own subset of active MLP parameters in order to characterize the uniqueness of this task.

- Joint Meta-learning. In this step, we re-train parameters of all three modules of each task using MAML again, but each private module is with its pruned MLP structure now. Also, similar tasks with similar pruned MLP structures are jointly trained in order to enrich the training data.

In the following, we will describe these two steps respectively as well as the gradient update of the whole dialogue model.

\subsection{Private Network Pruning}

After pre-training, dialogue models of different tasks remain the same parameters $\theta$, including $\theta^{s} / \theta^{p} / \theta^{g}$ in the shared/private/gating module. In this step, the private module with parameters $\theta^{p}$ will evolve into different structures with parameters $\theta_{i}^{p}$ to capture the task's unique characteristics.

First, we fine-tune the whole dialogue model of each task from the MAML initialization with its own training data and add an L-1 regularization on the parameters of the private module. The goal of L-1 regularization here is to make the parameters sparse such that only parameters beneficial to generate task-specific sentences are active.

Second, we apply an up-to-bottom strategy to prune the private MLP for each task. This is equal to selecting edges in the fully connected layers in the MLP. We do not prune the layers connected to the input and output of the MLP. For the rest layers, we start the pruning from the one closest to the output first. For the $l$-th layer, we consider layers above it $(>l)$ are closer to the output, and its lower layers $(<l)$ are closer to the input. When we process the $l$-th layer, its upper layers should already be pruned. We only keep edges of the current processed layer whose weight excels a certain threshold $\gamma$. If all edges in the $l$ layer connected to a node is pruned, all edges connected to this node in the $l-1$ layer will also be pruned. In this way, the parameters in private module $\theta^{p}$ differentiates into $|T|$ parameters $\theta_{i}^{p}$, where each $\theta_{i}^{p}$ is a subset of $\theta^{p}$. The pruning algorithm described above is illustrated in Algorithm 1.

\subsection{Joint Meta-learning}

So far, every task has a unique network structure in its private module. Now we jointly train the whole dialogue models of all tasks.

We start from the pre-trained MAML initialization again. For the shared and gating modules, all tasks share the same parameters, and they are trained with all training data. The private module, which is to capture the uniqueness of each task, is supposed to be trained on task-specific data. However, we do not have sufficient training data for each task in the few-shot setting, thus the private module may not be trained well. Fortunately, all private modules evolve from the same MLP structure, and similar tasks naturally share overlapped network structures, i.e. remaining edges after pruning are overlapped. This inspires us to train each edge in the private MLP by all training samples of tasks in which this edge is not pruned.

Concretely, we train the private MLP in this way: 


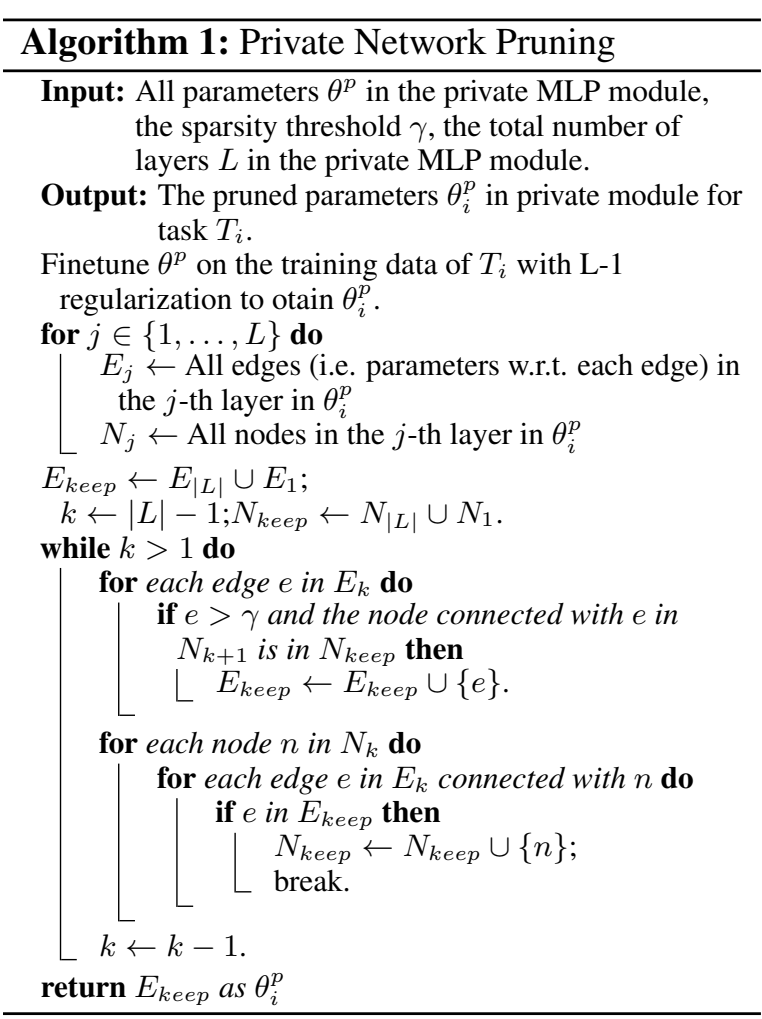

for each edge $e$ in the MLP, if it is active in more than one tasks, its corresponding parameters $\theta_{e}^{p}$ are updated on the data of all task $j$ 's, in which the edge is active, i.e. $\theta_{e}^{p} \in \theta_{j}^{p}$ :

$$
\theta_{e}^{\prime p}=\theta_{e}^{p}-\alpha \nabla_{\theta_{e}^{p}} \sum_{T_{j}: \theta_{e}^{p} \in \theta_{j}^{p}} \mathcal{L}_{D_{j}^{\text {train }}}\left(f\left(\theta_{j}^{p}\right)\right)
$$

where each $\theta_{i}^{p} / \theta_{i}^{\prime p}$ only contains the $\theta_{e}^{p} / \theta_{e}^{\prime p}$, s of all active edges in the $i$-th task.

During meta-testing, the loss is accumulated by the tasks that use the corresponding dialogue models, so $\theta^{p}$ is updated as,

$$
\theta^{p}=\theta^{p}-\beta \sum_{T_{i} \sim p(\mathcal{T})} \nabla_{\theta_{i}^{p}} \mathcal{L}_{D_{i}^{\text {valid }}}\left(f\left(\theta_{i}^{p}\right)\right)
$$

\subsection{Gradient Updates}

We summarize the gradient updates of the three modules in our proposed dialogue model during customized model training in Algorithm 2. For the shared and gating module, gradients are updated in the same way as MAML. The update of the private module is replaced by the above Eq. 4 and Eq. 5 introduced in joint meta-learning.

The loss function used to calculate the gradients in our model is the negative log-likelihood of generating the response $r$ given the input query $q$ as,

$$
\mathcal{L}=-\log p\left(r \mid q, \theta^{s}, \theta^{p}, \theta^{g}\right)
$$

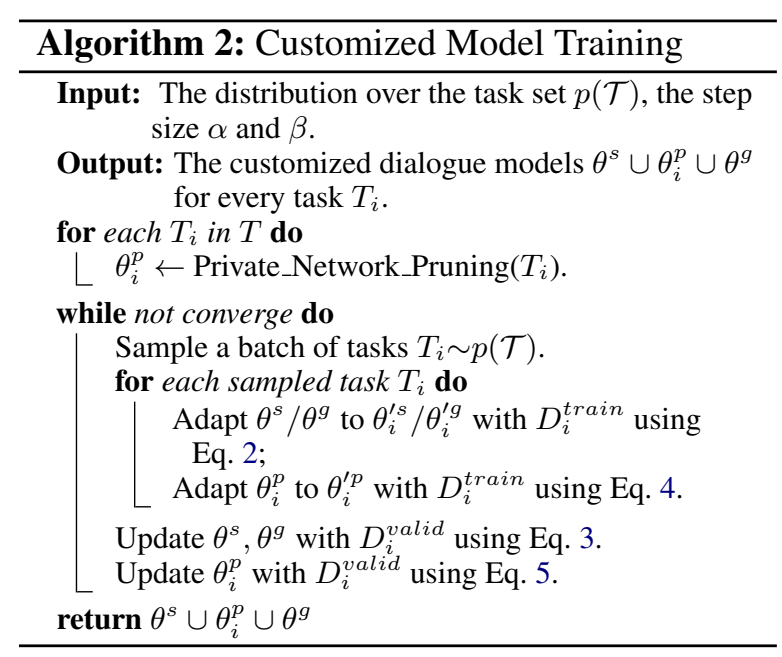

\section{Experiments}

\subsection{Datasets}

We perform experiments in Persona-chat [Madotto et al., 2019] and MojiTalk [Zhou and Wang, 2018], which are treated as few-shot dialogue generation tasks in previous work [Zhang et al., 2018; Madotto et al., 2019; Zhou and Wang, 2018; Zhou et al., 18]. Persona-chat has $1137 / 99 / 100$ users for training/validation/evaluation, and each user has 121 utterances on average. We follow the previous work [Madotto et al., 2019] and concatenate all the contextual utterances including the query as the input sequence. We regard building a dialogue model for a user as a task on this dataset. MojiTalk has 50/6/8 emojis for training/validation/evaluation. Each training/validation emoji has 1000 training samples on average, and each evaluation emoji has 155 samples on average. We regard generating responses with a designated emoji as a task. On both datasets, the data ratio for meta-training and meta-testing is $10: 1$.

\subsection{Implementation Details}

We implement our shared module based on the Seq2seq model with pre-trained Glove embedding [Pennington et al., 2014] and LSTM unit, and use a 4-layer MLP for the private module ${ }^{1}$. The dimension of word embedding, hidden state, and MLP's output are set to 300. In CMAML, we pretrain the model for 10 epochs and re-train each model for 5 steps to prune the private network. The L-1 weight in the re-training stage is 0.001 , and the threshold $\gamma$ is 0.05 . We follow other hyperparameter settings in Madotto et al. [2019].

\footnotetext{
${ }^{1}$ Code is available at https://github.com/zequnl/CMAML
} 


\subsection{Competing Methods}

- Pretrain-Only: We pre-train a unified dialogue generation model with data from all training tasks then directly test it on the testing tasks. We try three base generation models: the Seq2seq [Bahdanau et al., 2014] and the Speaker model [Li et al., 2016b] and the Seq2SPG proposed in Section3.1. Speaker incorporates the task (user/emoji) embeddings in the LSTM cell, and the task embeddings of testing tasks are random parameters in this setting.

- Finetune: We fine-tune the pre-trained models on each testing task, denoted as Seq2seq-F, Speaker-F and Seq2SPG-F.

- MAML [Madotto et al., 2019]: We apply the MAML algorithm on the base model Seq2seq and Seq2SPG, and note them as MAML-Seq2seq and $M A M L-S e q 2 S P G$. MAML-Seq2SPG uses the same base model as the proposed CMAML but does not apply the pruning algorithm, which helps to verify the effectiveness of the pruning algorithm and joint meta-learning. Note that We did not apply MAML on Speaker model as it shows no improvement comparing with Seq2seq.

- CMAML: We try two variants of our proposed algorithm. CMAML-Seq2SPG is our full model (equal to CMAML in previous sections), where the dialogue Seq2SPG is the base model and pruning algorithm is applied for customizing unique model structures for tasks. CMAML-Seq $2 S P^{\prime} G$ uses a different base model noted as Seq2SP'G, where the private module only takes the output of the shared module as the input. Pruning algorithm is also applied in private module for network customization.

\subsection{Evaluation Metrics}

Automatic Evaluation. We performed automatic evaluation metrics in three perspectives:

- Response quality/diversity: We use BLEU [Papineni et al., 2002] to measure the word overlap between the reference and the generated sentence; PPL, the negative logarithm of the generated sentence; Dist-1 [Li et al., 2016a; Song et al., 2017, 2018] to evaluate the response diversity, which calculates the ratio of distinct 1 -gram in all test generated responses.

- Task consistency: We use C score [Madotto et al., 2019] in Persona-chat, which uses a pre-trained natural language inference model to measure the response consistency with persona description, and E-acc [Zhou and Wang, 2018] in MojiTalk, which uses an emotion classifier to predict the correlation between a response and the designated emotion.

- Model difference: It is hard to measure the models ability of customization as we do not have the ground-truth model. Hence, we define the average model difference of pairwise tasks as the Diff Score of each method, and the model difference of a method before and after fine-tuning as $\Delta$ Score. The model difference between $T_{i}$ and $T_{j}$ is the Euclidean distance of their parameters normalized by their parameter count: $D\left(T_{i}, T_{j}\right)=\frac{\left\|\theta_{i}-\theta_{j}\right\|^{2}}{M}$. Here, $\theta_{i} / \theta_{j}$ includes all model parameters of this task, $M$ is the total parameter number of the model. A set of models that capture the unique characteristics of each task should be different from each other and will have a higher Diff score, indicating that a large Diff score is a sufficient condition for a strong customization ability. Similarly, a model that changes a lot for task specific adaptation during fine-tuning will achieve a higher $\Delta$ Score, indicating that $\Delta$ Score is also a sufficient condition for a good adaptation ability.

Human Evaluation. We invited 3 well-educated graduated students to annotate the 100 generated replies for each method. For each dataset, the annotators are requested to grade each response in terms of "quality" and "task consistency" (i.e. personality consistency in Persona-Chat and emoji consistency in MojiTalk) independently in three scales: 2 (for good), 1 (for fair) and 0 (for bad). "quality" measures the appropriateness of replies, and we refer 2 for fluent, highly consistent (between query and reply), and informativeness, 1 for few grammar mistakes, moderate consistent, and universal reply, and 0 for incomprehensible or unrelated topic. "task consistency" measures whether a reply is consistent with the characteristics of a certain task, and we refer 2 for highly consistent, 1 for no conflicted and 0 for contradicted. Notice that the user description (Persona dataset) and sentences with a certain emoji (Mojitalk dataset) are provided as the references. Volunteers, instead of authors, conduct the double-blind annotations on shuffled samples to avoid subjective bias.

\subsection{Overall Performance}

Quality/Diversity. In the Persona-chat dataset, Pretrain-Only methods provide the borderlines of all methods. In Pretrain-Only, Seq2SPG achieves the best performance in terms of both automatic and human measurements, indicating the appropri- 


\begin{tabular}{|c|c|c|c|c|c|c|c|c|}
\hline \multirow{2}{*}{ Method } & \multicolumn{2}{|c|}{ Human Evaluation } & \multicolumn{4}{|c|}{ Automatic Metrics } & \multicolumn{2}{|c|}{ Model Difference } \\
\hline & Quality & Task Consistency & PPL & BLEU & Dist-1 & C score/E-acc & Diff Score & $\Delta$ Score \\
\hline \multicolumn{9}{|l|}{ Persona-Chat } \\
\hline Seq2seq & 0.67 & 0.10 & 37.91 & 1.27 & 0.0019 & -0.16 & 0.00 & 0.00 \\
\hline Speaker & 0.85 & 0.10 & 40.17 & 1.25 & 0.0037 & -0.14 & 0.00 & 0.00 \\
\hline Seq2SPG & 0.67 & 0.03 & 36.46 & 1.41 & 0.0023 & -0.14 & 0.00 & 0.00 \\
\hline Seq2seq-F & 0.78 & 0.11 & 33.65 & 1.56 & 0.0046 & -0.05 & 17.97 & 9.19 \\
\hline Speaker-F & 0.87 & 0.25 & 35.61 & 1.52 & 0.0059 & 0.03 & 285.11 & 143.90 \\
\hline Seq2SPG-F & 0.7 & 0.07 & 32.68 & 1.54 & 0.0045 & -0.05 & 292.85 & 156.30 \\
\hline MAML-Seq2seq & 0.97 & 0.37 & 37.43 & 1.54 & 0.0087 & 0.14 & 134.01 & 67.79 \\
\hline MAML-Seq2SPG & 0.85 & 0.36 & 35.89 & 1.70 & 0.0074 & 0.16 & 401.28 & 198.90 \\
\hline CMAML-Seq2SP $^{\prime} \mathrm{G}$ & 0.98 & 0.58 & 37.32 & 1.43 & 0.0089 & 0.15 & 479.21 & 238.64 \\
\hline CMAML-Seq2SPG & 1.15 & 0.69 & 36.30 & 1.70 & 0.0097 & 0.18 & 514.44 & 263.82 \\
\hline \multicolumn{9}{|l|}{$\overline{\text { MojiTalk }}$} \\
\hline Seq2seq & 0.56 & 0.39 & 218.95 & 0.36 & 0.0342 & 0.73 & 0.00 & 0.00 \\
\hline Speaker & 0.38 & 0.26 & 418.96 & 0.19 & 0.0530 & 0.70 & 0.00 & 0.00 \\
\hline Seq2SPG & 0.77 & 0.46 & 158.74 & 0.64 & 0.0239 & 0.74 & 0.00 & 0.00 \\
\hline Seq2seq-F & 0.50 & 0.35 & 217.60 & 0.40 & 0.0326 & 0.72 & 15.96 & 8.88 \\
\hline Speaker-F & 0.39 & 0.25 & 403.92 & 0.21 & 0.0528 & 0.72 & 39.08 & 29.10 \\
\hline Seq2SPG-F & 0.76 & 0.47 & 157.92 & 0.65 & 0.0228 & 0.74 & 72.43 & 40.94 \\
\hline MAML-Seq2seq & 0.66 & 0.29 & 179.02 & 0.54 & 0.0109 & 0.70 & 183.05 & 117.09 \\
\hline MAML-Seq2SPG & 0.71 & 0.40 & 181.56 & 0.73 & 0.0246 & 0.74 & 306.40 & 176.31 \\
\hline CMAML-Seq2SP $^{\prime} \mathrm{G}$ & 0.64 & 0.32 & 172.92 & 0.76 & 0.0102 & 0.75 & 142.90 & 81.15 \\
\hline CMAML-Seq2SPG & 0.78 & 0.49 & 185.97 & 0.85 & 0.0210 & 0.77 & 345.42 & 190.64 \\
\hline
\end{tabular}

Table 1: Overall performance in Persona-chat (top) and MojiTalk (bottom) dataset in terms of quality (Human, Perplexity, BLEU), diversity (Dist-1), task consistency (Human, C score, E-acc), structure differences among tasks (Diff Score $\left.\left(\times 10^{-10}\right)\right)$, model change after adaptation $\left(\Delta\right.$ score $\left.\left(\times 10^{-10}\right)\right)$.

\begin{tabular}{|c|c|c|c|c|c|c|c|c|c|c|c|c|}
\hline \multirow{2}{*}{ Method } & \multicolumn{3}{|c|}{ 100-shot } & \multicolumn{3}{|c|}{ 110-shot } & \multicolumn{3}{|c|}{ Similar Users } & \multicolumn{3}{|c|}{ Dissimilar Users } \\
\hline & PPL & BLEU & C score & PPL & BLEU & C score & PPL & BLEU & C score & PPL & BLEU & C score \\
\hline Seq2seq & 38.13 & 1.19 & -0.11 & 37.58 & 1.29 & -0.15 & 76.54 & 1.49 & -0.03 & 42.87 & 1.10 & -0.10 \\
\hline Speaker & 40.95 & 1.02 & -0.25 & 42.59 & 1.27 & -0.06 & 162.44 & 0.65 & -0.09 & 46.86 & 1.11 & -0.13 \\
\hline Seq2SPG & 39.75 & 1.27 & -0.10 & 37.71 & 1.30 & -0.15 & 73.58 & 1.32 & -0.04 & 42.21 & 1.14 & -0.22 \\
\hline Seq2seq-F & 34.86 & 1.39 & -0.03 & 34.14 & 1.52 & -0.10 & 74.53 & 1.53 & -0.07 & 42.33 & 1.33 & -0.06 \\
\hline Speaker-F & 37.11 & 1.30 & -0.16 & 39.10 & 1.36 & -0.06 & 103.81 & 1.04 & 0.04 & 40.47 & 1.40 & 0.01 \\
\hline Seq2SPG-F & 37.19 & 1.31 & 0.00 & 37.00 & 1.33 & -0.15 & 70.15 & 1.44 & -0.04 & 36.22 & 1.35 & -0.05 \\
\hline MAML-Seq2seq & 36.94 & 1.47 & 0.03 & 37.20 & 1.53 & 0.07 & 83.17 & 1.52 & -0.08 & 39.67 & 1.34 & 0.06 \\
\hline MAML-Seq2SPG & 36.50 & 1.52 & 0.11 & 35.98 & 1.47 & 0.13 & 82.37 & 1.52 & -0.06 & 39.41 & 1.41 & 0.12 \\
\hline CMAML-Seq2SP $^{\prime} \mathrm{G}$ & 37.18 & 1.46 & 0.11 & 37.08 & 1.44 & 0.09 & 82.56 & 1.50 & 0.00 & 40.50 & 1.40 & 0.13 \\
\hline CMAML-Seq2SPG & 36.52 & 1.52 & 0.14 & 36.44 & 1.57 & 0.15 & 82.78 & 1.56 & -0.07 & 39.55 & 1.43 & 0.16 \\
\hline
\end{tabular}

Table 2: The performance on the Persona-chat dataset for impact factor analysis. The left figure is about the few-shot settings and the right is about the task similarity.

ateness of the proposed model structure. Finetune methods are better than Pretrain-Only methods in most cases. MAML methods have no better performance on BLEU scores than Finetune methods but have relatively higher Dist-1 scores. This indicates that MAML helps to boost response diversity. Enhanced with the proposed pruning algorithm, we can see great improvement for CMAML methods against all the competing methods on both quality and diversity measurements. Particularly, our full model CMAML-Seq2SPG shows clearly better performance and the reasons can be ascribed to two aspects: firstly, the proposed $S e q 2 S P G$ has a better model structure for our task and secondly, the pruning algorithm makes the models more likely to generate a user-coherent response.

Most of the performance of the competing methods in the MojiTalk dataset is similar to the Persona-chat dataset, while one difference is that Speaker achieves the highest Dist-1 score among all the methods. By carefully analyzing the gener- ated cases, we find all non-meta-learning methods (Pretrain-Only and Finetune) consistently produce random word sequences, which means they completely fail in the few-shot setting on this task. However, meta-learning-based methods survive.

Task Consistency. On both datasets, Finetune methods make no significant differences on $\mathrm{C}$ score, E-acc and Task Consistency when compared with Pretrain-Only methods, which means that simple fine-tuning is useless for improving the task consistency. All meta-learning methods including MAML and CMAML outperforms Finetune. Compared with MAML-Seq2seq and MAMLSeq2SPG, CMAML-Seq2SPG obtain 22.2\%/12.5\% and $11.8 \% / 5.6 \%$ improvement on $\mathrm{C}$ score and $\mathrm{E}-$ acc. It means that the private modules in $C M A M L$ $S e q 2 S P G$ are well pruned to better well describes the unique characteristics of each task.

We also observe that in MojiTalk, CMAML$S e q 2 S P G$ achieves good improvement compared with other baselines on the BLEU score but a lim- 
ited improvement on E-acc and task consistency score when compared with Persona-chat. This tells that when the training data is limited, the generative models tend to focus on the correctness of the response rather than the task consistency.

By jointly analyzing the response quality and task consistency measurement, we can easily draw the conclusion that the responses produced by our algorithm in CMAML-Seq2SPG not only is superior in response quality but also caters to the characteristics of the corresponding task.

Model Differences. Even though a high difference score among tasks does not indicate each model has captured its unique characteristics, a set of models that can capture the characteristics of themselves will have a higher different score. Hence, we present the difference scores of competing methods as a reference index. In Table 1, we can see that fine-tuning on non-meta-learning methods (Pretrain-Only and Finetune) does not boost the model differences between tasks. MAML helps to increase the model differences but is not as good as the proposed CMAML methods. CMAMLSeq2SPG achieves the highest model difference scores on two datasets as it distinguishes different tasks in both parameter and model structure level.

A higher $\Delta$ score of a method means its produced dialogue models are more easy to finetune. All non-meta-learning methods have so much lower $\triangle$ scores than MAML methods. $C M A M L$ Seq2SPG has the highest scores on both datasets, indicating that the active edges in the private module are more likely to be fine-tuned to better fit the corpus of the corresponding tasks. We also observe that $C M A M L-S e q 2 S P^{\prime} G$ has relatively low $\Delta$ scores, which indicates its base generation model Seq $2 S^{\prime} G$ is not as good as $S e q 2 S P G$.

\subsection{Impact Factors}

We further examine two factors that may have a great impact on the performance: the quantity of training data and the similarity among tasks.

Few-shot Settings. We only use Persona-chat dataset for analysis, because MojiTalk has too little data to further decrease. In Persona-chat, each user has 121 training samples on average, and we evaluate all the methods in a 100 and 110 samples setting (both in train and test) in Table 2 because all the methods tend to produce random sequences when each task contains less than 100 samples.

For non-meta-learning methods including

\begin{tabular}{l|l}
\hline Persona & $\begin{array}{l}\text { I also love vintage cars. } \\
\text { I am a pediatrician. } \\
\text { I love running and reading. }\end{array}$ \\
\hline \hline Query & $\begin{array}{l}\text { Singing karaoke is a talent of mine. } \\
\text { Do you sing too? }\end{array}$ \\
\hline Response & Not really. I am into running, books and old cars. \\
\hline \hline Seq2seq & $\begin{array}{l}\text { I do not have any pets. I do not have any pets. } \\
\text { Speaker }\end{array}$ \\
Seq2SPG & No, I do not. I do not have any. \\
\hline Seq2seq-F & I do not hot have any pets. I do not have any pets. \\
Speaker-F & No, I do not. I do not have any. \\
Seq2SPG-F & No, I do not have any pets. \\
\hline MAML-Seq2seq & $\begin{array}{l}\text { Yes I do. I am a nurse. } \\
\text { I like to listen to jazz and jazz . }\end{array}$ \\
MAML-Seq2SPG & Yes, I am a doctor. I am a pediatrician. \\
\hline CMAML-Seq2SP'G & Yes, I am a pediatrician. \\
CMAML-Seq2SPG & What do you do for a living? \\
\hline
\end{tabular}

Table 3: A case in Persona-chat dataset.

Pretrain-Only and Finetune, the quality scores improve as the quantity of training data increases, while the $\mathrm{C}$ scores almost remain the same as these methods are not sensitive to the differences among tasks. MAML methods have not changed too much on BLEU scores along with the data growth, but its $\mathrm{C}$ scores keep increasing. Both the BLEU score and C score of CMAML-Seq2SPG keep increasing with the data growth, and it always achieves the best performance among all the tasks. This proves that the customized generative models are suitable for the corresponding tasks and can always take the full potential of the training data.

Task Similarity. Again, we only use the Personachat dataset because we cannot define similarities among emojis. We construct two datasets: one contains 100 similar users and another contains 100 dissimilar users (both in train and test).

The performance of all the methods is close to each other in the similar-user setting. It means meta-learning-based methods have no advantage for similar tasks. In the dissimilar-users setting, CMAML-Seq2SPG performs best on the C score and BLEU. We draw a conclusion that user similarity influences the performance of our model. Compared to that in dissimilar-users setting, the BLEU in the similar-users setting is high, but the $\mathrm{C}$ score is low. The possible reason is that generative models do not distinguish similar tasks and regard all tasks as one task in training.

\subsection{Case Study}

We only present one case in the Persona-chat dataset due to the limited space in Table 3 . Pretrain-Only and Finetune methods produce general responses with less information. MAML methods tend to generate diverse responses as their initial parameters are easier to be finetuned. Even 
though the user profiles are not used for training, $C M A M L-S e q 2 S P G$ can quickly learn the persona information "pediatrician" from its training dialogues while other baselines can not. From another perspective, the pruned private module in CMAMLSeq2SPG can be regarded as a special memory that stores the task-specific information without explicit definition of memory cells.

\section{Conclusion}

In this paper, we address the problem of the fewshot dialogue generation. We propose CMAML, which is able to customize unique dialogue models for different tasks. CMAML introduces a private network for each task's dialogue model, whose structure will evolve during the training to better fit the characteristics of this task. The private module will only be trained on the corpora of the corresponding task and its similar tasks. The experiment results show that CMAML achieves the best performance in terms of response quality, diversity and task consistency. We also measure the model differences among tasks, and the results prove that CMAML produces diverse dialogue models for different tasks.

\section{Acknowledgement}

This paper is partially supported by National Key Research and Development Program of China with Grant No. 2018AAA0101900/2018AAA0101902, Beijing Municipal Commission of Science and Technology under Grant No. Z181100008918005, and the National Natural Science Foundation of China (NSFC Grant No. 61772039, No. 91646202, and No. 61876196).

\section{References}

Christoph Alt, Marc Hübner, and Leonhard Hennig. Fine-tuning pre-trained transformer language models to distantly supervised relation extraction. In ACL, pages 1388-1398, 2019.

Christoph Alt, Marc Hübner, and Leonhard Hennig. Fine-tuning pre-trained transformer language models to distantly supervised relation extraction. In ACL, pages 1388-1398, 2019.

Marcin Andrychowicz, Misha Denil, Sergio Gomez, Matthew W Hoffman, David Pfau, Tom Schaul, Brendan Shillingford, and Nando De Freitas. Learning to learn by gradient descent by gradient descent. In NeuralPS, pages 3981-3989, 2016.
Dzmitry Bahdanau, KyungHyun Cho, and Yoshua Bengio. Neural machine translation by jointly learning to align and translate. arXiv preprint arXiv:1409.0473, 2014.

Trapit Bansal, Rishikesh Jha, and Andrew McCallum. Learning to few-shot learn across diverse natural language classification tasks. arXiv preprint arXiv:1911.03863, 2019.

Andrew Brock, Theodore Lim, James Millar Ritchie, and Nicholas J Weston. Smash: One-shot model architecture search through hypernetworks. In ICLR, 2018.

Kyunghyun Cho, Bart van Merrienboer, Dzmitry Bahdanau, and Yoshua Bengio. On the properties of neural machine translation: Encoder-decoder approaches. In SSST-8, pages 103-111, 2014.

Jacob Devlin, Ming-Wei Chang, Kenton Lee, and Kristina Toutanova. Bert: Pre-training of deep bidirectional transformers for language understanding. arXiv preprint arXiv:1810.04805, 2018.

Chelsea Finn, Pieter Abbeel, and Sergey Levine. Model-agnostic meta-learning for fast adaptation of deep networks. In ICML, pages 1126-1135, 2017.

Sepp Hochreiter and Jürgen Schmidhuber. Long shortterm memory. Neural computation, 9(8):17351780, 1997.

Jeremy Howard and Sebastian Ruder. Universal language model fine-tuning for text classification. arXiv preprint arXiv:1801.06146, 2018.

Po-Sen Huang, Chenglong Wang, Rishabh Singh, Wen tau Yih, and Xiaodong He. Natural language to structured query generation via meta-learning. In NAACL-HLT, pages 732-738, 2018.

Xiang Jiang, Mohammad Havaei, Gabriel Chartrand, Hassan Chouaib, Thomas Vincent, Andrew Jesson, Nicolas Chapados, and Stan Matwin. Attentive taskagnostic meta-learning for few-shot text classification. arXiv preprint arXiv: 1805.07722, 2018.

Nikita Kitaev Steven Cao Dan Klein. Multilingual constituency parsing with self-attention and pre-training. In $A C L$, pages 3499-3505, 2019.

Jiwei Li, Michel Galley, Chris Brockett, Jianfeng Gao, and Bill Dolan. A diversity-promoting objective function for neural conversation models. In $N A A C L$, pages 110-119, 2016.

Jiwei Li, Michel Galley, Chris Brockett, Georgios Spithourakis, Jianfeng Gao, and Bill Dolan. A personabased neural conversation model. In $A C L$, pages 994-1003, 2016.

Luchen Liu, Zequn Liu, Haoxian Wu, Zichang Wang, Jianhao Shen, Yiping Song, and Ming Zhang. Multitask learning via adaptation to similar tasks for mortality prediction of diverse rare diseases. In arXiv preprint arXiv:2004.05318, 2020. 
Andrea Madotto, Zhaojiang Lin, Chien-Sheng Wu, and Pascale Fung. Personalizing dialogue agents via meta-learning. In ACL, pages 5454-5459, 2019.

Fei Mi, Minlie Huang, Jiyong Zhang, and Boi Faltings. Meta-learning for low-resource natural language generation in task-oriented dialogue systems In IJCAI, pages 3151-3157, 2019.

Tong Niu and Mohit Bansal. Adversarial oversensitivity and over-stability strategies for dialogue models. In CoNLL, pages 486-496, 2018.

Abiola Obamuyide and Andreas Vlachos. Metalearning improves lifelong relation extraction. In $A C L$, pages 224-229, 2019.

Abiola Obamuyide and Andreas Vlachos. Modelagnostic meta-learning for relation classification with limited supervision. In $A C L$, pages 5873-5879, 2019.

Kishore Papineni, Salim Roukos, Todd Ward, and WeiJing Zhu. Bleu: a method for automatic evaluation of machine translation. In $A C L$, pages $311-318$, 2002 .

Jeffrey Pennington, Richard Socher, and Christopher Manning. Glove: Global vectors for word representation. In EMNLP, pages 1532-1543, 2014.

Matthew E. Peters, Mark Neumann, Mohit Iyyer, Matt Gardner, Christopher Clark, Kenton Lee, and Luke Zettlemoyer. Deep contextualized word representations. arXiv preprint arXiv:1802.05365, 2018.

Kun Qian and Zhou Yu. Domain adaptive dialog generation via meta learning. In $A C L$, pages 2639-2649, 2019.

Alec Radford, Karthik Narasimhan, Tim Salimans, and Ilya Sutskever. Improving language understanding by generative pre-training. URL: https://s3-us-west-2. amazonaws. com/openaiassets/researchcovers/languageunsupervised/language understanding paper. pdf, 2018.

Adam Santoro, Sergey Bartunov, Matthew Botvinick, Daan Wierstra, and Timothy Lillicrap. Metalearning with memory-augmented neural networks. In ICML, pages 1842-1850, 2016.

Jake Snell, Kevin Swersky, and Richard Zemel. Prototypical networks for few-shot learning. In NeuralPS, pages 4077-4087, 2017.

Yiping Song, Zhiliang Tian, Dongyan Zhao, Ming Zhang, and Rui Yan. Diversifying neural conversation model with maximal marginal relevance. In IJCNLP, pages 169-174, 2017.

Yiping Song, Rui Yan, Yansong Feng, Yaoyuan Zhang, Dongyan Zhao, and Ming Zhang. Towards a neural conversation model with diversity net using determinantal point processes. In $A A A I$, pages 5932-5939, 2018.
Qianru Sun, Yaoyao Liu, Tat-Seng Chua, and Bernt Schiele. Meta-transfer learning for few-shot learning. In CVPR, pages 403-412, 2019.

Flood Sung, Yongxin Yang, Li Zhang, Tao Xiang, Philip HS Torr, and Timothy M Hospedales. Learning to compare: Relation network for few-shot learning. In $C V P R$, pages 1199-1208, 2018.

Ilya Sutskever, Oriol Vinyals, and Quoc V Le. Sequence to sequence learning with neural networks. In NeuralPS, pages 3104-3112, 2014.

Zhiliang Tian, Rui Yan, Lili Mou, Yiping Song, Yansong Feng, and Dongyan Zhao. How to make context more useful? an empirical study on contextaware neural conversational models. In $A C L$, volume 2, pages 231-236, 2017.

Oriol Vinyals, Charles Blundell, Timothy Lillicrap, Daan Wierstra, et al. Matching networks for one shot learning. In NeuralPS, pages 3630-3638, 2016.

Haoyu Wang, Ming Tan, Mo Yu, Shiyu Chang, Dakuo Wang, Kun $\mathrm{Xu}$, Xiaoxiao Guo, and Saloni Potdar. Extracting multiple-relations in one-pass with pretrained transformers. In $A C L$, pages 1371-1377, 2019.

Haoyu Wang, Ming Tan, Mo Yu, Shiyu Chang, Dakuo Wang, Kun $\mathrm{Xu}$, Xiaoxiao Guo, and Saloni Potdar. Extracting multiple-relations in one-pass with pretrained transformers. In $A C L$, pages 1371-1377, 2019.

Zhi-Xiu Ye and Zhen-Hua Ling. Multi-level matching and aggregation network for few-shot relation classification. In $A C L$, pages 2872-2881, 2019.

Mo Yu, Xiaoxiao Guo, Jinfeng Yi, Shiyu Chang, Saloni Potdar, Yu Cheng, Gerald Tesauro, Haoyu Wang, and Bowen Zhou. Diverse few-shot text classification with multiple metrics. In NAACL-HLT, pages 1206-2015, 2019

Saizheng Zhang, Emily Dinan, Jack Urbanek, Arthur Szlam, Douwe Kiela, and Jason Weston. Personalizing dialogue agents: I have a dog, do you have pets too? In $A C L$, pages 2204-2213, 2018.

Xianda Zhou and William Yang Wang. Mojitalk: Generating emotional responses at scale. In $A C L$, pages 1128-1137, 2018.

Hao Zhou, Minlie Huang, Tianyang Zhang, Xiaoyan Zhu, and Bing Liu. Emotional chatting machine: Emotional conversation generation with internal and external memory. In $A A A I$, pages 730-738, 18 .

Luisa Zintgraf, Kyriacos Shiarli, Vitaly Kurin, Katja Hofmann, and Shimon Whiteson. Fast context adaptation via meta-learning. In ICML, pages 76937702, 2019. 